\title{
Double Retroperitoneal Hydatid Localization: A Case Report
}

\author{
Ramzi Mejri ${ }^{1}$, Kays Chaker ${ }^{2}$, Mokhtar Bibi ${ }^{2}$, Sami Ben Rhouma ${ }^{2}$, Yassine Nouira ${ }^{2}$ \\ ${ }^{1}$ Department of Urology, Hospital Mongi Slim La Marsa, La Marsa, Tunisia \\ ${ }^{2}$ Department of Urology, La Rabta Hospital, Tunis, Tunisia
}

\begin{abstract}
The hydatid cyst is a parasitic pathology which is endemic in Tunisia and presents a public health problem. Hydatid cysts located in the retroperitoneum, especially around or in the kidney, are rare and only represent $5 \%$ of visceral locations. The kidney is the most commonly affected organ of the urinary tract. The psoas muscle is an uncommon location and not less than 70 cases have been cited. We report the case of an unusual presentation of a right kidney hydatid cyst associated with a psoas muscle location. To our knowledge, this association of double retroperitoneal location has not been reported in the literature.
\end{abstract}

Received August 2, 2021

Revised August 20, 2021

Accepted August 27, 2021

Corresponding author:

Ramzi Mejri

Email: ramzimejrimed@yahoo.fr

https://orcid.org/0000-0002-1913-0740

Key Words: Hydatid cyst, Kidney, Muscular, Psoas

Hydatidosis is a zoonosis secondary to the development of the larval or hydatid form of Echinococcus granulosis (a very small tapeworm or dog Echinococcus) in humans. The hydatid cyst is a parasitic pathology which is endemic in Tunisia and presents a public health problem with an incidence of 12.6/100,000 inhabitants. ${ }^{1}$ The liver and lung represent the most frequent locations. Hydatid cysts located in the retroperitoneum, especially around or in the kidney, are rare and only represent $5 \%$ of visceral locations. The kidney is the most commonly affected organ of the urinary tract, although this involvement is rare. ${ }^{2}$ The hydatid cyst rarely affects the striated musculature, in particular the psoas muscle. A frequency of muscle involvement of around $3 \%$ has been reported in the literature ${ }^{3}$ and no less than 70 cases of hydatid cysts of the psoas have been mentioned in scientific reviews. ${ }^{4}$ The selectivity of the proximal muscles could be explained by the importance of blood flow. We report an original and uncommon case of an unusual presentation of a hydatid cyst of the right renal associated with a retroperitoneal location dependent on the psoas muscle. To our knowledge, this association of double retroperitoneal location of hydatid cyst has not been reported in the literature.

\section{CASE REPORT}

A 49-year-old woman presented to our urological surgery unit complaining of the presence of pain in the right flank associated with hydaturia. The patient also reports the notion of few episodes of vomiting from last 5 days, anorexia, asthenia, and weight loss. In her medical history, we can cite well-balanced diabetes mellitus, hypertension, and chronic back pain. She denied any context or family history of malignancy. The patient, originally 
from Kasserine, had no medical history of hydatid vomiting or close contact with stray dogs or other animals. Clinical examination shows a large mass which fills the right lumbar fossa, firm, sensitive and which is palpable by bimanual palpation of the kidney. Laboratory test showed a normal level of inflammatory markers and a normal renal function. An ultrasound of the urinary tract showed a right kidney increased in size, site of a voluminous upper medullary and polar formation,

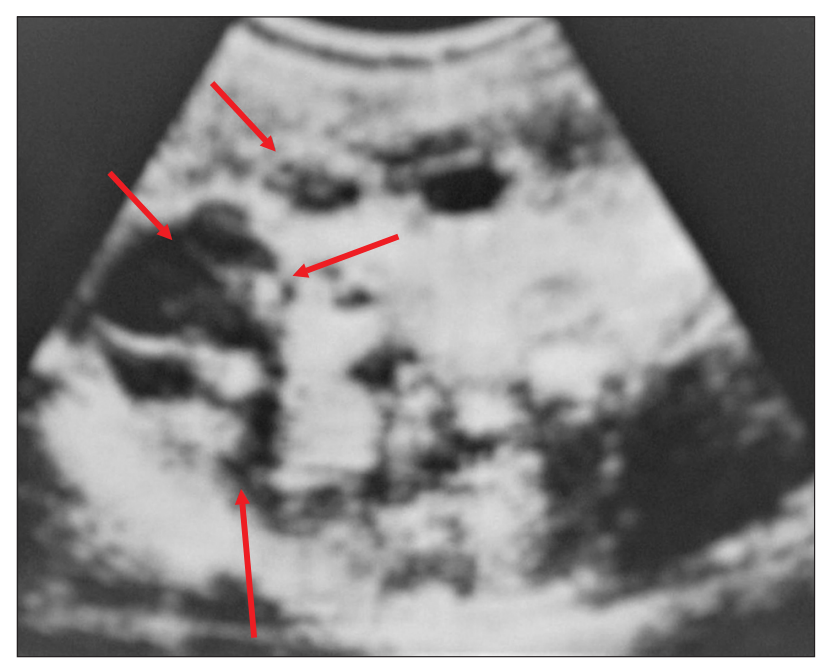

Fig. 1. Ultrasound of the urinary tract: heterogeneous echogenic formation (arrows) of the upper pole of the right kidney.

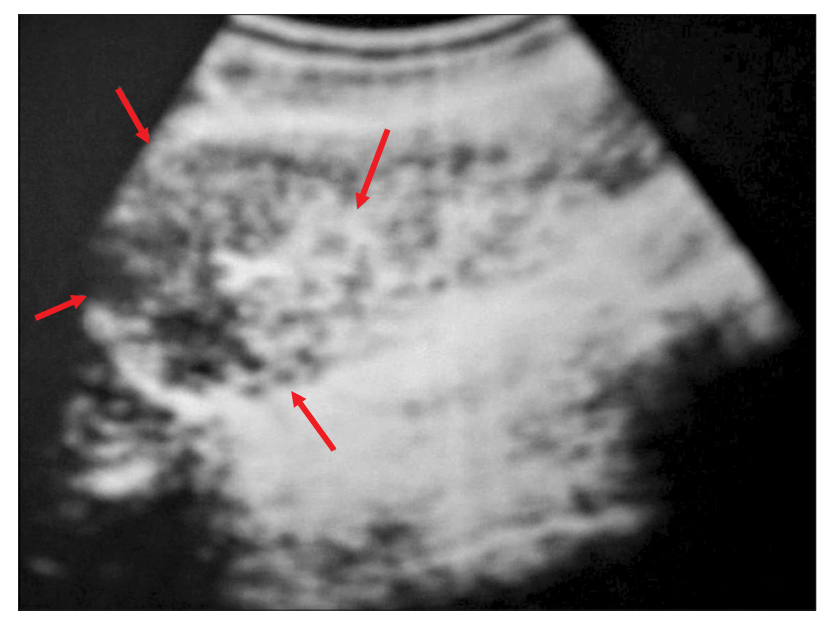

Fig. 2. Ultrasound of the urinary tract: heterogeneous formation along the right psoas (arrows). polylobed, heterogeneous with its own echogenic wall (Fig. 1). Another oblong heterogeneous formation of $13 \mathrm{~cm}$ along the right psoas was found (Fig. 2). Intravenous urography showed a right renal mass syndrome with repression and stretching of the middle and lower calyceal group (Fig. 3). The ultrasound data was confirmed by contrast enhanced computed tomography scan of the upper abdomen. Computed tomography revealed a hypodense multicystic mass at the level

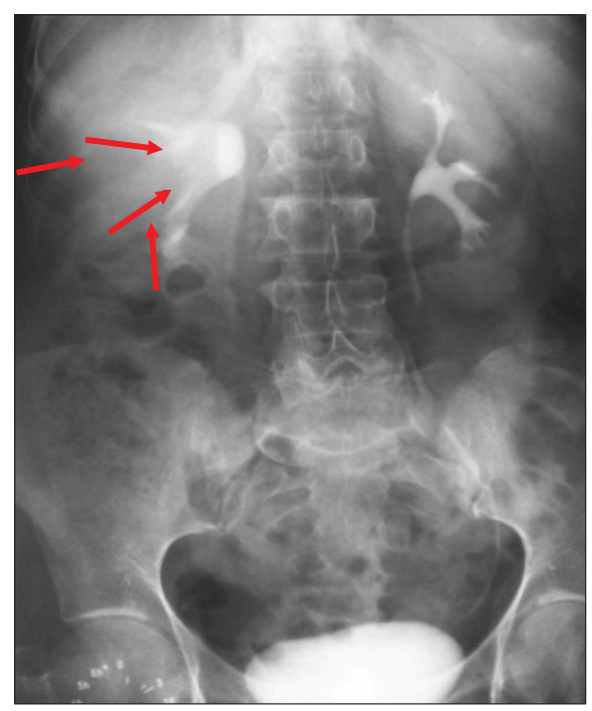

Fig. 3. Intravenous urography: right medio-renal mass syndrome (arrows).

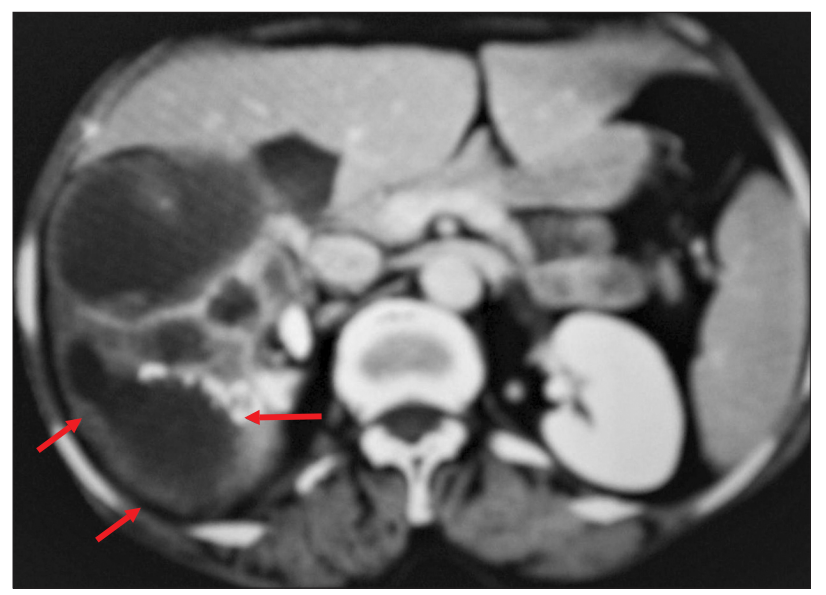

Fig. 4. Computed tomography: right renal calcified multicystic mass (arrows). 


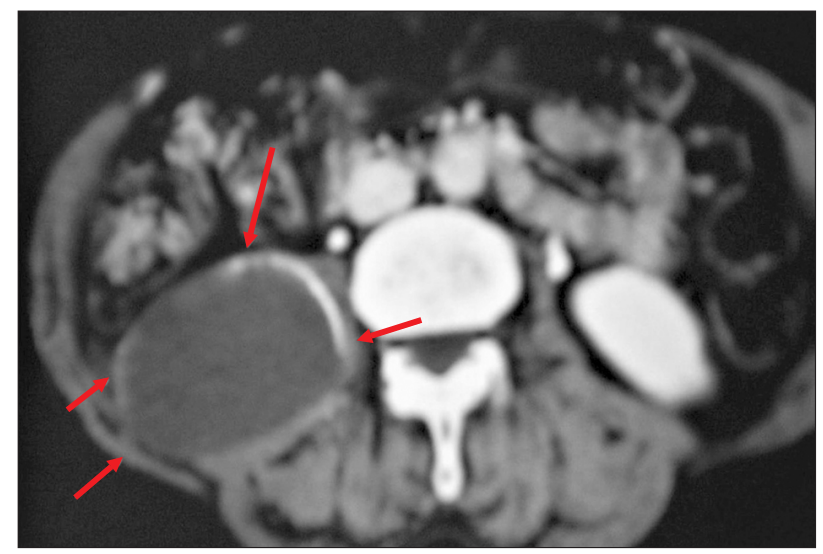

Fig. 5. Computed tomography: cystic mass of the right psoas (arrows).

of the right renal superior pole with thick calcified walls. These radiological data characterize hydatid disease. Another cystic lesion of the same nature in contact with the lower pole of the right kidney was found (Figs. 4, 5). The chest $\mathrm{x}$-ray is normal. The hydatid serology is negative. The patient was managed by open surgery: lombotomy. Resection of the protruding dome of the renal cyst and a pericystectomy for the cyst of the right psoas were performed. Following resection of the protruding dome, proligerous membrane was clearly observed. No incidents were reported during the postoperative period. The patient was discharged from the hospital on day 4 after a favorable clinical outcome. The anatomopathological study concluded in a hydatid cyst of the kidney and psoas (microscopic exploration revealed the presence of protoscolex alive in the intracystic fluid). The patient is regularly monitored in the outpatient department, he was in good physical and mental health, his renal function was normal. After a 3-year follow-up, no hydatid recurrence was reported.

\section{DISCUSSION}

Hydatidosis has a worldwide distribution and represents a serious health problem in endemic regions such as Mediterranean countries. It is a zoonosis caused by the development of the human larva of the taenia Echinococcus granulosus. The embryonic eggs, eliminated from the external environment with the dog's bowel movements, are accidentally ingested and enter the intestinal wall. The larvae migrate into the small bowel wall and the mesenteric circulation, getting filtered by the liver, which is the reason why hepatic infection is so common. The second site of filtration is the lung, which is involved in $15 \%$ of the cases. ${ }^{5}$ However, almost any tissue in the body can be infected through hematogenous spread. ${ }^{6}$ Primary hydatid cysts develop in the retroperitoneal tissue, without apparent connection, remain an exceptional entity. Soft tissue involvement is not classic. It is reported in $0.5 \%$ to $4.7 \%$ of cases and mainly affects the musculature of the neck and lower limbs. This is due to the degree of vascularization of the tissues and to the muscular contractions which would prevent the development of the larva. The high lactic acid content of the muscle has been reported to prevent hydatid growth. ${ }^{7}$ In hydatid endemic countries, all locations of hydatid cyst are possible and retroperitoneal localization is rare. Renal hydatid cysts are extremely rare, most often unilateral. The pathogenesis of renal Echinococcus is speculative. It is not known how the hydatid embryo can primarily affect the kidneys, but it is assumed that it passes through the portal system and the retroperitoneal lymphatic system without lodging there. Kidney hydatid can be complicated to rupture. Cystic rupture of the collecting system causes hydatiduria, observed in only $10 \%$ to $20 \%$ of renal hydatics as is the case in our patient. Cyst rupture can occur in the retroperitonial space and thus be grafted onto any retroperitoneal organ such as the psoas muscle. Concerning our patient, we can assume a retroperitoneal haematic 
transplant of the larvae of taenia in the renal and the psoas muscle. This hypothesis is strongly suggested by the fact that no rupture of the renal hydatid cyst in the retroperitoneal space has been reported.

The diagnosis of hydatid cyst of the psoas muscle, often difficult. Clinical examination may show an abdominal mass, located in the iliac or lumbar region, renitent, fixed to the deep plane. Some cysts can be revealed by complications such as nerve, urinary or vascular compression, or by a superinfection by the hematogenous route which may cause sometimes severe sepsis. Hydatidosis serologies are less and less performed because of their low reliability. These tests are often negative or inadequate for definitive diagnosis if the cyst is intact, calcified, or sterile. ${ }^{8}$ Imaging is essential for diagnosis and pretreatment assessment. Ultrasound is a harmless first-line examination. In deep locations such as the psoas, the interest of a computed tomography study is necessary. The scanner allows an easy diagnosis with a more precise topographic assessment.

The treatment is surgical. The principle of hydatid cyst surgery is to neutralise and evacuate the contents of the cyst while avoiding dissemination into the peritoneal cavity. Thus, for all these reasons, the retroperitoneal approach is preferable. As for the renal location, the principle of surgery in the event of localization of the psoas is to eradicate the entire cyst. Total pericystectomy is the treatment of choice. Due to the close relationship of the cyst with the ureter or iliac pedicle, partial pericystectomy is therefore the safest therapeutic option.

\section{CONFLICT OF INTEREST}

The authors claim no conflicts of interest.

\section{ORCID}

Ramzi Mejri, https://orcid.org/0000-0002-1913-0740

Kays Chaker, https://orcid.org/0000-0003-0193-3972

Mokhtar Bibi, https://orcid.org/0000-0001-7023-0731

Sami Ben Rhouma, https://orcid.org/0000-0002-2328-3897

Yassine Nouira, https://orcid.org/0000-0002-8684-7266

\section{REFERENCES}

1. Ben Ismail I, Zenaidi H, Rebii S, Zoghlami A. Primary hydatic cyst of the fallopian tube. IDCases 2020;20:e00790.

2. Garg R, Nahar U, Mandal AK. A rare case of primary renal hydatid cyst. Ann Parasitol 2015;61:12931.

3. Bedioui H, Nouira K, Daghfous A, Ammous A, Ayari $\mathrm{H}$, Rebai W, et al. Primary hydatid cyst of the psoas muscle: description of 9 cases in Tunisia and review of the literature. Med Trop (Mars) 2008;68: 261-6.

4. Mseddi M, Mtaoumi M, Dahmene J, Ben Hamida R, Siala A, Moula T, et al. Kyste hydatique musculaire: à propos de 11 cas. Rev Chir Orthop Reparatrice Appar Mot 2005;91:267-71. (French).

5. Bandyopadhyay A, Khatua S, Das S, Bose K, Konar K. A rare case of primary renal hydatid cyst presenting with hydatiduria. J Parasit Dis 2015;39:57780.

6. Gupta M, Singh R, Lehl SS. Isolated renal hydatid cyst in a diabetic postmenopausal female. J Midlife Health 2015;6:134-6.

7. Soufi M, Lahlou MK, Messrouri R, Benamr S, Essadel A, Mdaghri J, et al. Kyste hydatique du psoas: à propos de deux cas. J Radiol 2010;91:1292-4.

8. Wani I, Wani RA, Alam Wani K. Primary hydatid of psoas muscle. Urol Ann 2010;2:89. 